\title{
Detection Limits of DLS and UV-Vis Spectroscopy in Characterization of Polydisperse Nanoparticles Colloids
}

\author{
Emilia Tomaszewska, ${ }^{1}$ Katarzyna Soliwoda, ${ }^{1}$ Kinga Kadziola, ${ }^{1}$ Beata Tkacz-Szczesna, ${ }^{1}$ \\ Grzegorz Celichowski, ${ }^{1}$ Michal Cichomski, ${ }^{1}$ Witold Szmaja, ${ }^{2}$ and Jaroslaw Grobelny ${ }^{1}$ \\ ${ }^{1}$ Department of Materials Technology and Chemistry, Faculty of Chemistry, University of Lodz, \\ Pomorska 163, 90-236 Lodz, Poland \\ ${ }^{2}$ Department of Solid State Physics, Faculty of Physics and Applied Informatics, University of Lodz, \\ Pomorska 149/153, 90-236 Lodz, Poland \\ Correspondence should be addressed to Jaroslaw Grobelny; jgrobel@uni.lodz.pl
}

Received 15 April 2013; Revised 17 June 2013; Accepted 18 June 2013

Academic Editor: William W. Yu

Copyright (C) 2013 Emilia Tomaszewska et al. This is an open access article distributed under the Creative Commons Attribution License, which permits unrestricted use, distribution, and reproduction in any medium, provided the original work is properly cited.

\begin{abstract}
Dynamic light scattering is a method that depends on the interaction of light with particles. This method can be used for measurements of narrow particle size distributions especially in the range of 2-500 $\mathrm{nm}$. Sample polydispersity can distort the results, and we could not see the real populations of particles because big particles presented in the sample can screen smaller ones. Although the theory and mathematical basics of DLS technique are already well known, little has been done to determine its limits experimentally. The size and size distribution of artificially prepared polydisperse silver nanoparticles (NPs) colloids were studied using dynamic light scattering (DLS) and ultraviolet-visible (UV-Vis) spectroscopy. Polydisperse colloids were prepared based on the mixture of chemically synthesized monodisperse colloids well characterized by atomic force microscopy (AFM), transmission electron microscopy (TEM), DLS, and UV-Vis spectroscopy. Analysis of the DLS results obtained for polydisperse colloids reveals that several percent of the volume content of bigger NPs could screen completely the presence of smaller ones. The presented results could be extremely important from nanoparticles metrology point of view and should help to understand experimental data especially for the one who works with DLS and/or UV-Vis only.
\end{abstract}

\section{Introduction}

Nanoparticles (NPs) of noble metals, especially silver (Ag) and gold $(\mathrm{Au}) \mathrm{NPs}$, attract much attention in various fields. This is because of the unique properties of nanoscale objects, which are completely different from bulk materials, coatings, and single atoms. Applications or potential applications of NPs are not only diverse but also interdisciplinary and are related to material science, electronics, optics, and biomedicine. Noble metal NPs (in particular Ag NPs) can facilitate important advances in detection, diagnosis, and treatment of human cancers as well as delivery of drug and gene to malignant cells [1,2]. By exploiting the unique optical and electronic properties of Au NPs, several new methods for ultrasensitive detection of DNA, RNA, and proteins have been developed [3-7].
Currently, many applications of silver NPs are related to their versatile antibacterial activity against a broad spectrum of bacteria without releasing toxic biocides. It should be noted that depending on the concentration and size silver nanoparticles can be considered as nontoxic as well as toxic [8-10]. Ag NPs are environmentally friendly antibacterial materials and therefore are used in many cosmetic products and medical applications such as hydrogel dressings [11]. Metal NPs are also promising for surface-enhanced Raman scattering (SERS) applications in detection and analysis of molecules. The enhancement factor can be strong enough that the technique allows detection of a single molecule [12-14].

As noble metal NPs are widely used in many applications, it is important to synthesize high-quality materials. In certain applications it is also important to prepare colloids with high 
concentrations. The size and size distribution are becoming extremely significant when quantum-sized effects are used to control material properties. Therefore, the control and complete analysis of the average particle size and narrow size distribution is essential to use NPs in many applications $[15,16]$.

There are a number of methods for nanoparticles size characterization, for example, scanning/transmission electron microscopy (SEM/TEM), atomic force microscopy (AFM) analytical ultracentrifugation (AUC), dynamic light scattering (DLS), and flow field fractionation (FFF). It is also possible to calculate particles size with the use of $\mathrm{X}$ ray diffraction (XRD) patterns as well as with the shift of the band gap absorption in the UV-Vis spectrum $[17,18]$. In case of the above-mentioned method [19-23], the particle size distribution is measured in dispersion or after drying the sample. The detailed description of the above-mentioned methods is beyond the scope of this work and will not be described.

There is a range of methods available for the particle size determination, and users should remember that precise characterization of particles requires the usage of few of them. Moreover, it is essential to know about the strengths and weaknesses of applied method for nanoparticles characterization in order to recognize the limits; for example, FFF and AUC methods suffer from artifacts like too small particle diameter because of the particle charge [24]. In case of TEM, it is not always obvious to recognize grain boundaries in aggregates. The main advantage of microscopic techniques is that it is possible to get the information about the morphology and the size of particles at the same time, but the preparation of samples for analysis is crucial (e.g., NPs must be deposited on the well-characterized substrate). Moreover, sample preparation is often time consuming, requires high precision and the use of appropriate reagents [25-27]. Microscopic techniques can also be problematic in case of the polydisperse samples because of possibility of particles aggregation or sample fractionation during drying.

Among the techniques of nanoparticles characterization the most commonly used are DLS [23, 28, 29] and UV-Vis spectroscopy [30-33]. The theory and mathematical basics of DLS and UV-Vis techniques are already well known [34, 35]. DLS measures the light scattered from the laser that passes through a colloid. Next, the modulation of the scattered light intensity as a function of time is analyzed, and the hydrodynamic size of particles can be determined [36, 37]. In case of UV-Vis spectroscopy, the intensity of light that is passing through the sample is measured. Nanoparticles have optical properties that are very sensitive on size, shape, agglomeration, and concentration changes. The unique optical properties of metal nanoparticles are a consequence of the collective oscillations of conduction electrons, which excited by electromagnetic radiation are called surface plasmon polariton resonances (SPPR) [35]. Those changes have an influence on the refractive index next to the nanoparticles surface; thus it is possible to characterize nanomaterials using UV-Vis spectroscopy. DLS and UV-Vis spectroscopy are fast and easy to operate techniques for particles characterization, especially for colloidal suspensions [38, 39]. There are several advantages of DLS and UV-Vis techniques: simplicity, sensitivity and selectivity to NPs, short time of measurement, and what is more, the calibration is not required. Therefore, these techniques are increasingly used for NPs characterization in many fields of science and industry $[22,23,40]$. Although the DLS technique is widely used for particles characterization, there are some problems in case of measuring samples with large-size distribution or multimodal distributions [23, 29]. If the measured colloid is monodisperse, the mean diameter of NPs can be determined using DLS technique. In case of polydisperse colloids, there is a risk that during the DLS measurement small objects can be screened by bigger ones and will not even be seen at all. By far, little has been done to determine the detection limits of DLS and UV-Vis techniques experimentally. The main aim of this study was to detect limits of widely used DLS and UV-Vis spectroscopy in characterization of polydisperse nanoparticles, colloids (Ag NPs colloids as mixture of nanoparticles with different sizes, $10 \mathrm{~nm}$, and $55 \mathrm{~nm} ; 10 \mathrm{~nm}$ and $80 \mathrm{~nm}$ ) and to determine the possibilities to observe small objects $(10 \mathrm{~nm})$ in the presence of large ones (55 $\mathrm{nm}$ and $80 \mathrm{~nm}$ ).

To investigate the detection limits of DLS and UV-Vis techniques colloids with the nanoparticles size about: 10, 55 and $80 \mathrm{~nm}$ were used. Selected colloids have nanoparticles sizes below $100 \mathrm{~nm}$ which is related to the definition of nanomaterials defined by the European Commission. The selected colloids give the possibility to perform high-quality and reliable measurements (the lower limit of particles size for DLS measurements is about $10 \mathrm{~nm}$ ). The size of nanoparticles about $55 \mathrm{~nm}$ is in the middle of the selected limits (10 $\mathrm{nm}$ and $80 \mathrm{~nm})$.

DLS and UV-Vis spectroscopy as well as AFM and TEM were used to characterize the monodisperse silver NPs. The colloids were intentionally mixed in appropriate volume ratio, and mixtures (polydisperse colloids) were studied using the DLS and UV-Vis techniques.

There are works that compare results from different NPs size measurement techniques $[29,41,42]$, but in general the researchers do not apply to polydisperse metallic NPs with the size up to $100 \mathrm{~nm}$. To the authors' knowledge, the present paper reports the results of such an investigation for the first time.

\section{Materials and Methods}

2.1. Synthesis of Monodisperse Silver Nanoparticles. To prepare the polydisperse colloids, it was necessary to synthesize and characterize monodisperse Ag NPs colloids. Ag NPs were obtained in three different sizes: $10 \mathrm{~nm}, 55 \mathrm{~nm}$, and $80 \mathrm{~nm}$. For the synthesis of NPs [43], the following reagents were used: silver nitrate $\left(\mathrm{AgNO}_{3}\right.$, purity $99.999 \%$, obtained from Sigma-Aldrich), and sodium citrate $\left(\mathrm{C}_{6} \mathrm{H}_{5} \mathrm{Na}_{3} \mathrm{O}_{7} \cdot 2 \mathrm{H}_{2} \mathrm{O}\right.$, purity $99.0 \%$, obtained from Sigma-Aldrich), tannic acid $\left(\mathrm{C}_{76} \mathrm{H}_{52} \mathrm{O}_{46}\right.$, obtained from Fluka), sodium borohydride $\left(\mathrm{NaBH}_{4}\right.$, purity $\geq 96 \%$, obtained from Sigma-Aldrich). Deionized water was obtained from Deionizer Millipore Simplicity UV system. 
After the synthesis, the amount of silver ions was determined using flame atomic adsorption spectrometry (AAS, Varian, Spectra 300). The amount of silver ion was less than $0.5 \mathrm{ppm}$.

2.1.1. Synthesis of $10 \mathrm{~nm}$ Ag NPs (Sample 1). Silver NPs with an average diameter of $10 \mathrm{~nm}$ were prepared as follows: into $95.5 \mathrm{~g}$ of aqueous silver nitrate solution at the concentration of $0.017 \%$, set on a mechanical stirrer, a mixture of sodium citrate $(4.2 \mathrm{~g}, 4 \%)$ and tannic acid $(0.63 \mathrm{~g}, 5 \%)$ was added. Immediately after mixing reagents, $0.7 \mathrm{~g}$ of solution of sodium borohydride, at the concentration of $2 \%$, was added. After the addition of reductants, the color of the solution changed into brown. The whole mixture was vigorously stirred for $15 \mathrm{~min}$. Final concentration of Ag in colloids was 100 ppm.

2.1.2. Synthesis of $55 \mathrm{~nm} \mathrm{Ag} \mathrm{NPs} \mathrm{(Sample} \mathrm{2).} \mathrm{Silver} \mathrm{NPs} \mathrm{with}$ an average diameter of $55 \mathrm{~nm}(100 \mathrm{ppm})$ were prepared by reduction of $\mathrm{AgNO}_{3}$ by sodium citrate and tannic acid. An aqueous solution of $\mathrm{AgNO}_{3}(48.85 \mathrm{~g}, 0.016 \%)$ was heated to boil and stirred under reflux. Next the mixture of an aqueous solution of sodium citrate $(0.52 \mathrm{~g}, 4 \%)$ and an aqueous solution of tannic acid $(0.63 \mathrm{~g}, 5 \%)$ was added. After the addition of the reductants the color of the solution changed into yellow, which indicated the formation of the silver NPs. The solution was vigorously stirred under reflux for additional $15 \mathrm{~min}$, and after that time the suspension was cooled to room temperature. Final concentration of $\mathrm{Ag}$ in colloids was $100 \mathrm{ppm}$.

2.1.3. Synthesis of $80 \mathrm{~nm}$ Ag NPs (Sample 3). Silver NPs with an average diameter of $80 \mathrm{~nm}$ were prepared as described above, but with the different amount of silver nitrate ( $492.7 \mathrm{~g}$, $0.048 \%$ ), sodium citrate $(4.2 \mathrm{~g}, 15 \%)$, and tannic acid (3.15 g, $30 \%)$. After synthesis, the solution was dissolved to final concentration of $\mathrm{Ag}$ in colloids which was $100 \mathrm{ppm}$.

2.2. Measurement Techniques. The resulting monodisperse NPs of silver colloids (samples 1-3) were examined using the AFM, TEM, DLS, and UV-Vis techniques.

2.2.1. Atomic Force Microscopy. AFM measurements of NPs were performed with the use of a commercial AFM system (Solver P47, NT-MDT), operating in air under ambient conditions. Images were typically obtained in the tapping mode using a rectangular silicon nitride cantilever (NSC $35 / \mathrm{Si}_{3} \mathrm{~N}_{4} /$ AlBS, MikroMasch). The composition of the 1 , 2 , and 3 colloids varies. In case of samples 1, 2, and 3, the nanoparticles surface stabilizer is different, so that it was necessary to use a different surface modifier for the more effective adsorption of nanoparticles on the substrate. Hence, different substrates (mica or silicon wafer) for AFM measurements were used. Moreover, the usage of a different modifier has a negligible effect on the measured size of NPs. In order to carry out AFM measurements, colloids of silver NPs were deposited on mica (sample 1) or silicon substrates (samples 2 and 3) using the procedure described in [25].
2.2.2. Transmission Electron Microscopy. The size and shape of Ag NPs (samples 1 and 2) were evaluated by a high resolution transmission electron microscope, JEOL JEM 1200 $\mathrm{EX}$, at an accelerating voltage of $120 \mathrm{kV}$. TEM samples of the silver NPs (samples 1, 2, and 3) were prepared by placing of the product solution onto the carbon-coated copper grids, allowing the solvent to evaporate in air.

2.2.3. Dynamic Light Scattering. The size and size distribution of particles in the colloids were measured using a Nano ZS zetasizer system (Malvern Instruments). Measurement parameters were as follows: a laser wavelength of $633 \mathrm{~nm}$ (He-Ne), a scattering angle of $173^{\circ}$ (fixed-without changing possibility), a measurement temperature of $25^{\circ} \mathrm{C}$, a medium viscosity of $0.8872 \mathrm{mPa} \cdot \mathrm{s}$ and a medium refractive index of 1.330 , and material refractive index of 0.200 . Before DLS measurement, the colloid was passed through a $0.2 \mu \mathrm{m}$ polyvinylidene fluoride (PVDF) membrane. The sample was loaded into quartz microcuvette, and five measurements were performed, for which the mean result was recorded. DLS studies were carried out in two modes: general purpose mode (with normal resolution) and multiple narrow mode (with high resolution).

2.2.4. UV-Vis Spectroscopy. UV-Vis spectra were recorded using as a light source versatile lamps optimized for the visible-near infrared Vis-NIR (360-2000 nm), Ocean Optics, HL-2000 (tungsten halogen light sources). To collect UVVis spectra, the USB2000 + detector (miniature fiber optic spectrometer) was used. Before measurement, the samples were diluted one hundred times. The presented results were obtained by averaging 1000 of single measurements.

2.3. Polydisperse Sample Preparation. The prepared and wellcharacterized monodisperse colloids were mixed together in appropriate volume ratios. To examine the sensitivity/resolution of DLS and UV-Vis techniques, the mixtures of monodisperse colloids with the size of 10,55 , and $80 \mathrm{~nm}$ were measured. The list of initial colloids and polydisperse samples prepared by the mixing of appropriate monodisperse colloids is presented in Table 1 .

The DLS and UV-Vis characterization was carried out for the samples 4-8 and 16-20. Additional measurements using UV-Vis spectroscopy were performed for the samples 9-15 and $21-27$.

\section{Results and Discussion}

3.1. Monodisperse Silver Nanoparticles. The colloids prepared as monodisperse silver NPs were measured using DLS. The size measured in DLS technique is the hydrodynamic diameter of the theoretical sphere that diffuses with the same speed as the measured nanoparticle. This size is not only connected with the metallic core of the nanoparticles (like it is in case of microscopic techniques when measuring the size of nanoparticles) but it is also influenced with all substances adsorbed on the surface of the nanoparticles (e.g., stabilizers) 
TABLE 1: Summary of tested samples. The numbers indicate the percentage of the volume for silver NPs of a particular size.

\begin{tabular}{|c|c|c|c|c|}
\hline \multirow{2}{*}{ Sample number } & \multicolumn{3}{|c|}{ Percentage volume [\%] } & \multirow{2}{*}{ Presented results } \\
\hline & $10 \mathrm{~nm}$ AgNPs & $55 \mathrm{~nm}$ AgNPs & $80 \mathrm{~nm}$ AgNPs & \\
\hline 1 & 100 & - & - & DLS, AFM, TEM, UV-Vis \\
\hline 2 & - & 100 & - & DLS, AFM, TEM, UV-Vis \\
\hline 3 & - & - & 100 & DLS, AFM, TEM, UV-Vis \\
\hline 4 & 99 & 1 & - & DLS, UV-Vis \\
\hline 5 & 98 & 2 & - & DLS, UV-Vis \\
\hline 6 & 97 & 3 & - & DLS, UV-Vis \\
\hline 7 & 96 & 4 & - & DLS, UV-Vis \\
\hline 8 & 95 & 5 & - & DLS, UV-Vis \\
\hline 9 & 90 & 10 & - & UV-Vis \\
\hline 10 & 80 & 20 & - & UV-Vis \\
\hline 11 & 70 & 30 & - & $\mathrm{UV}$-Vis \\
\hline 12 & 60 & 40 & & UV-Vis \\
\hline 13 & 50 & 50 & - & UV-Vis \\
\hline 14 & 40 & 60 & - & UV-Vis \\
\hline 15 & 20 & 80 & - & UV-Vis \\
\hline 16 & 99 & - & 1 & DLS, UV-Vis \\
\hline 17 & 98 & - & 2 & DLS, UV-Vis \\
\hline 18 & 97 & - & 3 & DLS, UV-Vis \\
\hline 19 & 96 & - & 4 & DLS, UV-Vis \\
\hline 20 & 95 & - & 5 & DLS, UV-Vis \\
\hline 21 & 90 & - & 10 & UV-Vis \\
\hline 22 & 80 & - & 20 & UV-Vis \\
\hline 23 & 70 & - & 30 & UV-Vis \\
\hline 24 & 60 & - & 40 & UV-Vis \\
\hline 25 & 50 & - & 50 & UV-Vis \\
\hline 26 & 40 & - & 60 & $\mathrm{UV}$-Vis \\
\hline 27 & 20 & - & 80 & UV-Vis \\
\hline
\end{tabular}

and the thickness of the electrical double layer (solvation shell), moving along with the particle. The thickness of the electrical double layer and its influence on the measured size of nanoparticles depend on the substances present in the colloid and on the surface of nanoparticles. As a consequence, the size measured in DLS technique is bigger in comparison with macroscopic techniques. The results obtained for the samples 1-3 are shown in Figure 1. Analyses of obtained results indicate that in the investigated colloids monodisperse NPs with size $(10 \pm 5) \mathrm{nm}$ (Figure 1(a)), (55 \pm 9) nm (Figure $1(\mathrm{~b})$ ), and $(80 \pm 20) \mathrm{nm}$ (Figure 1(c)) are present. In all cases, measurements uncertainties were calculated as the standard deviation. Measurements parameters were as follows: dispersant $\mathrm{RI}=1,330$; viscosity $=0,8872 \mathrm{cP}$; temperature $=25,0^{\circ} \mathrm{C}$; attenuator was set up automatically and ranged from 6 to 9. In case of all monodisperse AgNPs, the count rate was between 160 and 400 kcps. Polydispersity index (PdI) for monodisperse silver nanoparticles was smaller than 0,140 .

Figure 2 shows AFM images of Ag NPs (samples 1-3) homogeneously distributed on the different surfaces. Analysis of these images confirms that the silver NPs are monodisperse. The diameters of the obtained NPs were $(8 \pm 3) \mathrm{nm}$,
$(38 \pm 6) \mathrm{nm}$ and $(55 \pm 9) \mathrm{nm}$ for the samples 1,2 , and 3 , respectively. The average sizes of NPs were determined by measuring at least one hundred objects and creating a histogram. AFM images show that the investigated objects are monodisperse, without aggregates or agglomerates. The particles are characterized by a narrow size distribution around the mean value.

Additionally, TEM was also used to determine the average size of silver NPs. TEM images of sample 1 (Figure $3(\mathrm{a})$ ), sample 2 (Figure 3(b)), and sample 3 (Figure 3(c)) were recorded in order to verify the presence of monodisperse NPs. The diameters of nanoparticles were measured using Motic Plus 2.0 software. The population of analyzed nanoparticles was about 100 for AFM and TEM measurements. Next, the size distribution histogram was created, and for each data set, the mean size and standard deviation were calculated. The study allowed determining the average sizes of NPs to be $(10 \pm 2) \mathrm{nm},(41 \pm 5) \mathrm{nm}$, and $(61 \pm 11)$ $\mathrm{nm}$ for the samples 1, 2, and 3, respectively. Agglomerated nanoparticles seen on TEM images are connected with the sample preparation for measurements. In case of sample preparation for TEM measurements, it is not possible to 


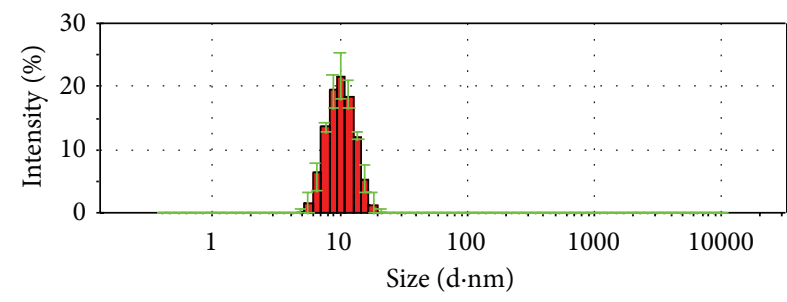

(a)

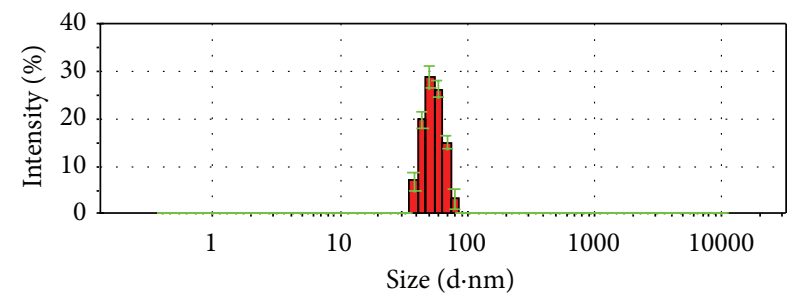

(b)

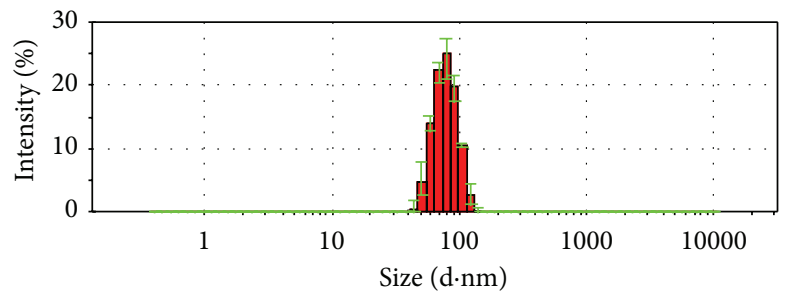

(c)

FIGURE 1: Size distribution of Ag NPs measured by the DLS technique for (a) sample 1, (b) sample 2, and (c) sample 3.

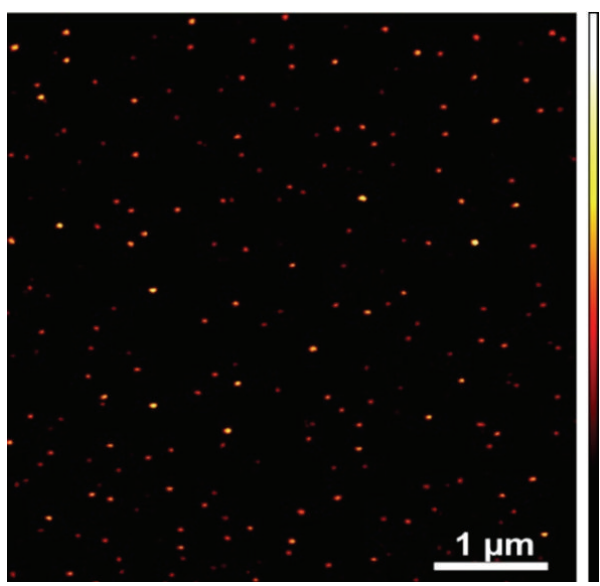

(a)

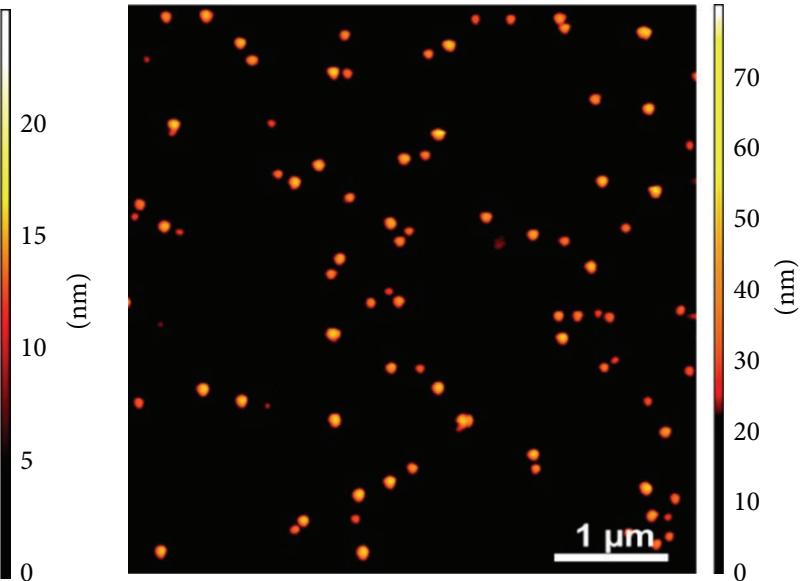

(b)

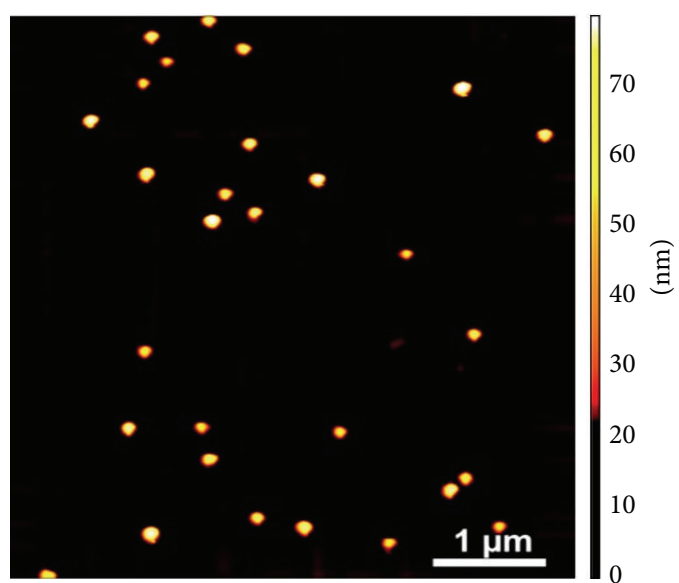

(c)

FIGURE 2: AFM images of monodisperse silver NPs for (a) sample 1 deposited on mica substrate, (b) sample 2 deposited on silicon substrate, and (c) sample 3 deposited on silicon substrate. 


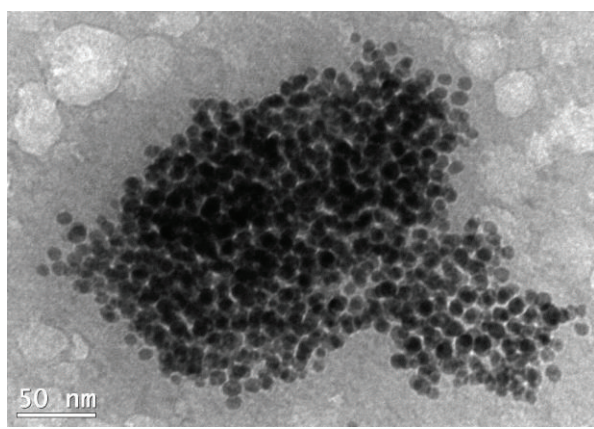

(a)

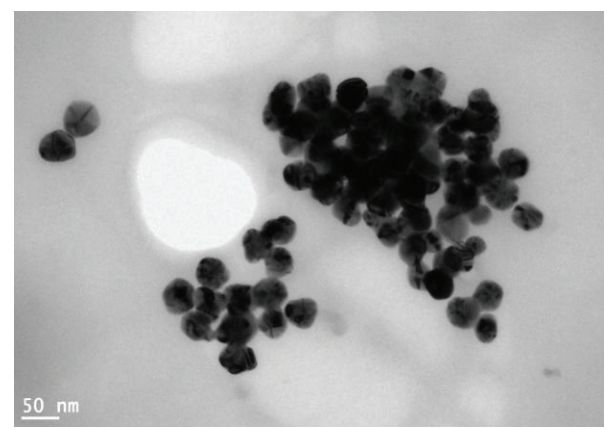

(b)

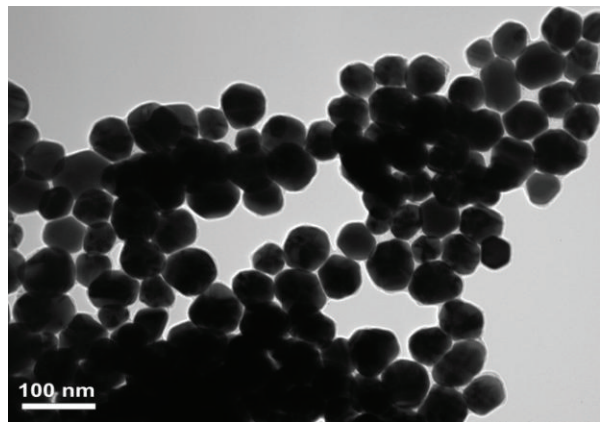

(c)

FIGURE 3: TEM images of monodisperse Ag NPs for (a) sample 1, (b) sample 2, and (c) sample 3.

modify the substrate surface (copper grid with a thin carbon coating). As a consequence, it may happen that some accumulated nanoparticles can be seen. However, AFM and DLS measurements revealed that colloids (sample 1, 2, and 3) are monodisperse.

For monodisperse colloids, measurements with the use of UV-Vis spectroscopy were also performed. The absorption peaks are located at wavelengths of $407 \mathrm{~nm}, 427 \mathrm{~nm}$, and $439 \mathrm{~nm}$ for the samples 1, 2, and 3, respectively.

It should be noted that differences in the size of NPs were determined by the use of different techniques. This is not caused by measurement error, but in fact by the specificity of each technique. TEM and AFM measure the geometric size of the NPs deposited on the surface, so the results from these two techniques are similar. In case of the DLS technique, the hydrodynamic size is measured. This size is characterized for the ball model, which has the same diffusion coefficient as a measured NP. As a result, the size of measured NPs can differ from that determined by the AFM/TEM techniques. In general, each presented measurement method shows that only monodisperse particles are present in the investigated colloids.

3.2. Polydisperse Colloids of Silver Nanoparticles. The DLS results of the polydisperse colloids obtained by mixing monodisperse $10 \mathrm{~nm}$ and $55 \mathrm{~nm} \mathrm{Ag} \mathrm{NPs} \mathrm{(samples} \mathrm{4-8)} \mathrm{are}$ shown in Figure 4. Similar studies using DLS have been carried out for the polydisperse colloids obtained by mixing
$10 \mathrm{~nm}$ with $80 \mathrm{~nm} \mathrm{Ag} \mathrm{NPs} \mathrm{(samples} \mathrm{16-20),} \mathrm{and} \mathrm{the} \mathrm{obtained}$ results are presented in Figure 5. It can be observed that when the volume of bigger particles increases the signal from small particles decreases. This is a consequence of the fact that the ability of a particle to scatter light is proportional to its diameter to the sixth power [41]. The peak coming from $10 \mathrm{~nm} \mathrm{Ag} \mathrm{NPs} \mathrm{disappears} \mathrm{completely} \mathrm{at} \mathrm{the} \mathrm{95 \%} \mathrm{of}$ the content of these particles in the colloid (Figure 4(e)). In the considered case, the intensity of light scattered by larger particles ( $55 \mathrm{~nm}$ or $80 \mathrm{~nm} \mathrm{Ag} \mathrm{NPs)} \mathrm{totally} \mathrm{conceals}$ the signal from smaller ones (10 nm Ag NPs). And as a consequence, such a result can wrongly suggest that only monodisperse particles $55 \mathrm{~nm}$ (or $80 \mathrm{~nm}$ ) in size are present in the colloid.

The characterization results of the optical properties of the polydisperse silver colloids obtained by mixing monodisperse $10 \mathrm{~nm}$ and $55 \mathrm{~nm}(10 \mathrm{~nm}$ and $80 \mathrm{~nm}) \mathrm{Ag}$ NPs in the appropriate volume ratio are shown in Figure 6(a) (Figure 6(b)).

It can be seen that the maximum absorption wavelength increases with the increasing of larger size Ag NPs percentage volume. Finally the maximum absorption peak is shifted to a wavelength of $427 \mathrm{~nm}(439 \mathrm{~nm})$ when only particles with the size of $55 \mathrm{~nm}(80 \mathrm{~nm})$ are present in the solution. Such a redshift is characteristic for an increased NPs size [43, 44]. The position of absorption maximum of polydisperse colloid does not give information about the size of nanoparticles, because it is not possible to observe peaks separation form specific populations in these colloids. 


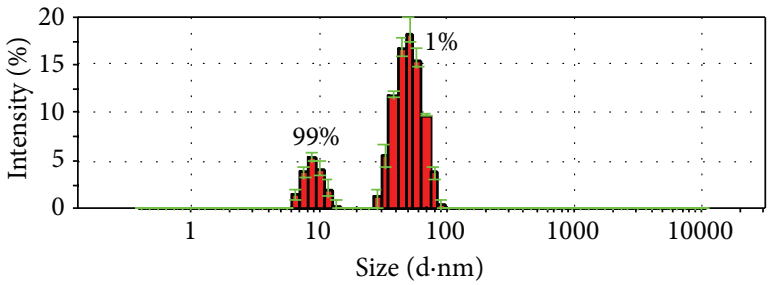

(a)

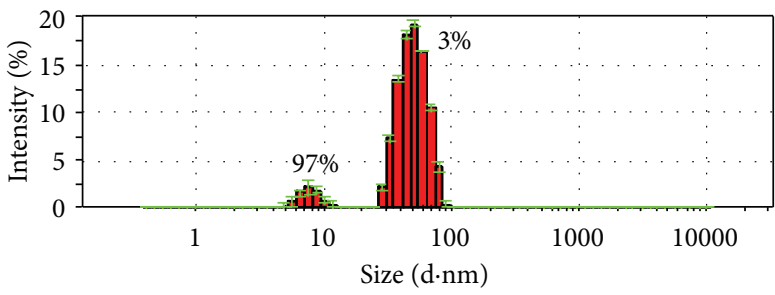

(c)

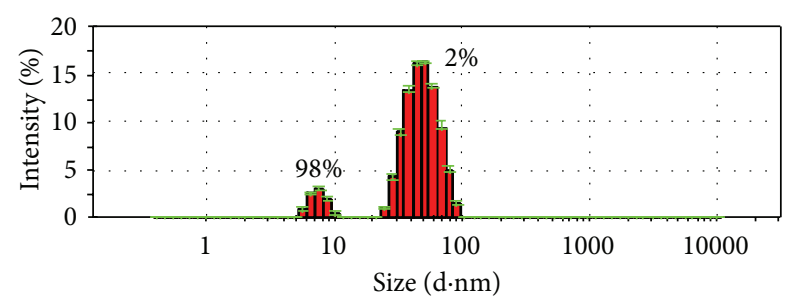

(b)

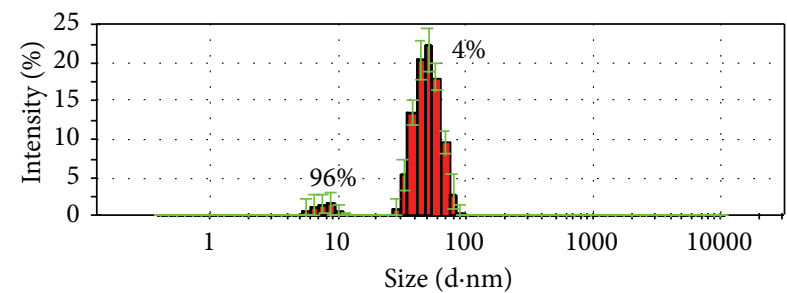

(d)

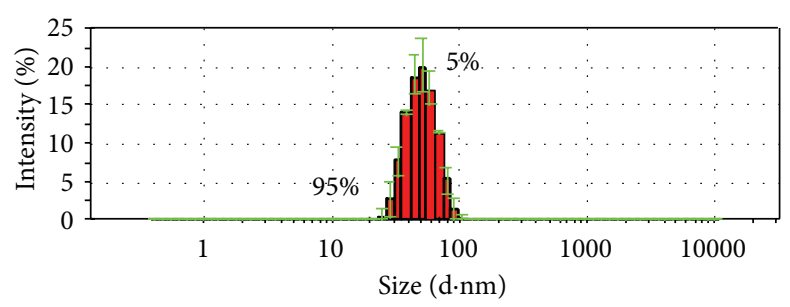

(e)

Figure 4: Size distribution of silver NPs measured by the DLS technique for mixtures of monodisperse $10 \mathrm{~nm}$ and $55 \mathrm{~nm}$ Ag NPs for (a) sample 4, (b) sample 5, (c) sample 6, (d) sample 7, and (e) sample 8. The numbers close to the histogram bars indicate the percentage of the volume for Ag NPs of a particular size in the analyzed mixture.

\section{Conclusions}

Measurements of the artificially prepared polydisperse colloids were performed using the DLS and UV-Vis spectroscopy techniques. Based on the DLS results, it can be concluded that the detection of smaller NPs in the presence of several percent of bigger ones (mixed together as polydisperse colloids) seems to be in fact very difficult.

Simple calculations show that the number of particles with the size of $10 \mathrm{~nm}$ in the tested mixtures could be even three orders of magnitude larger than that of $55 \mathrm{~nm}$ particles and four orders of magnitude larger than that of $80 \mathrm{~nm}$ particles (under the same silver mass concentration per volume unit). However, the light scatter from bigger AgNPs is so intense that the scatter light coming from smaller AgNPs is concealed. Consequently it is not possible to detect the signal coming from 95\% of smaller AgNPs in the presence of $5 \%$ bigger AuNPs. Depending on the combination of monodisperse colloids applied to prepare artificial polydisperse colloid, the detection limits of DLS and UV-Vis techniques can vary. In case of UV-Vis spectroscopy the separation of peaks for NPs of different sizes was not observed. Measurements of polydisperse colloids showed only the shift of the peak maximum compared with monodisperse samples. Hence, UV-Vis should not be used as a routine method to quantitatively examine particle size but there are a lot of researches where only UV-Vis is used to quantitatively examine particle size $[45,46]$.

There are other measurement techniques where using a lot more complicated equipment is possible to observe specific populations of nanoparticles in polydisperse colloids [47]. However, the main advantage of DLS and UV-Vis is that these techniques are easy to perform as well as quick and cheap. Nevertheless, the present paper clearly shows that while carrying out the synthesis of NPs, one should be very careful while interpreting the results obtained with the mentioned techniques. The obtained results are not unambiguous and an inexperienced investigator may be misled. One has to be aware that in the case of DLS or UV-Vis spectroscopy investigation of polydisperse NPs, it is necessary to make also study by much more reliable techniques such as AFM or TEM/SEM.

\section{Acknowledgments}

Scientific work was supported by funds for science in 20112014 allocation for the cofounded international project. This work was supported by the Polish Ministry of Science and Higher Education within Research Grant no. NN 507350435. 


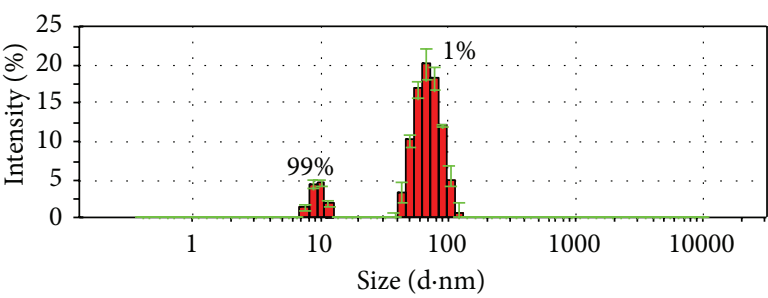

(a)

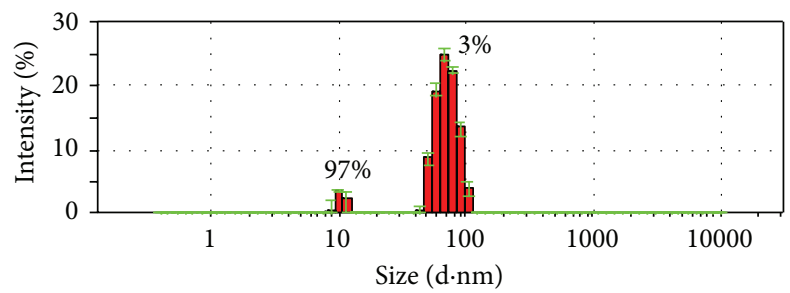

(c)

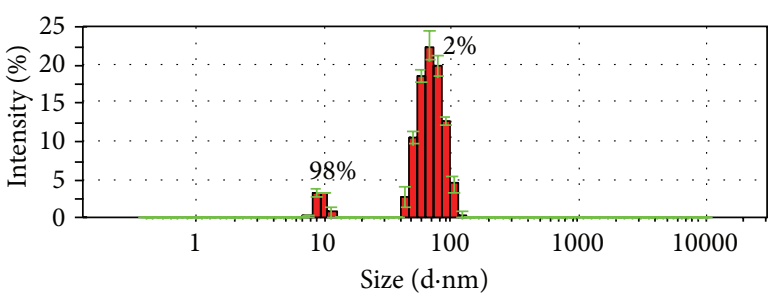

(b)

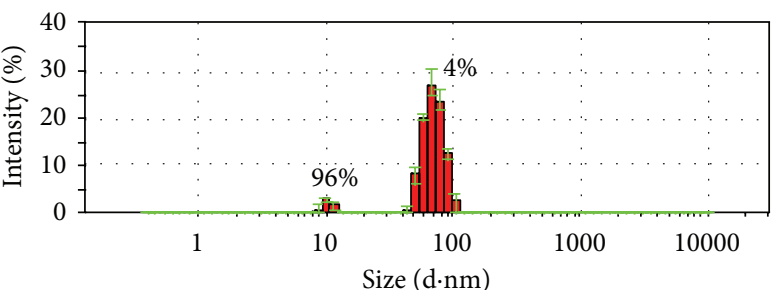

(d)

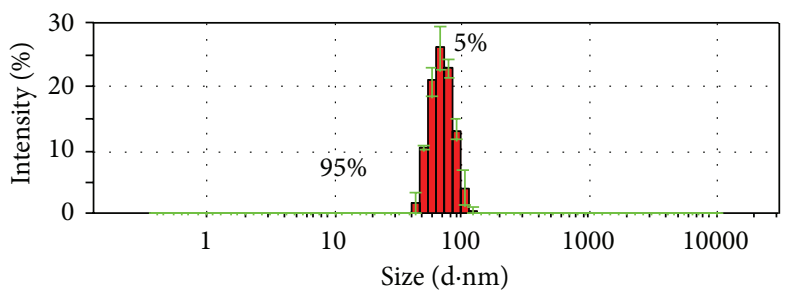

(e)

FIgURE 5: Size distribution of silver NPs measured by the DLS technique for mixtures of monodisperse $10 \mathrm{~nm}$ and $80 \mathrm{~nm}$ Ag NPs for (a) sample 16, (b) sample 17, (c) sample 18, (d) sample 19, and (e) sample 20. The numbers close to the histogram bars indicate the percentage of the volume for Ag NPs of a particular size in the analyzed mixture.

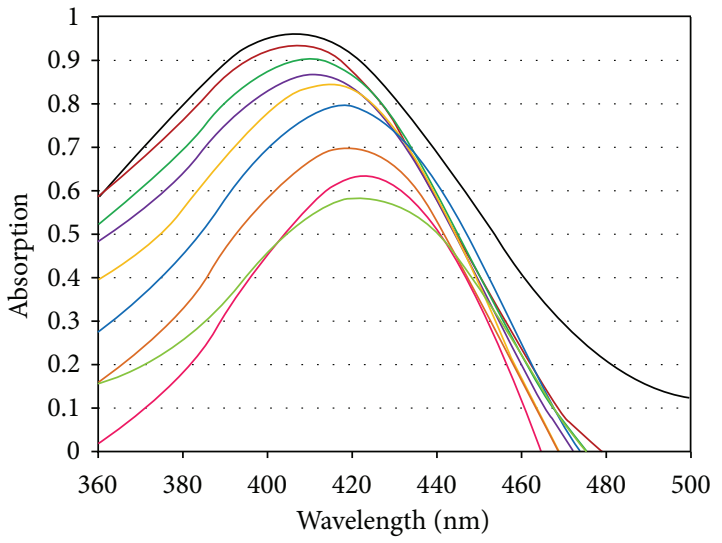

Sample 1

Sample 9

Sample 10

Sample 11

- Sample 12

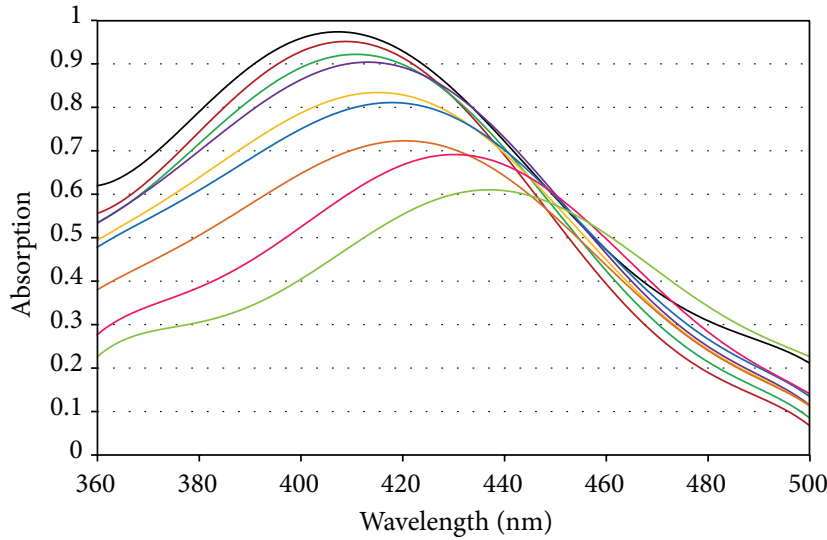

Sample 1

Sample 21

Sample 22

Sample 23

Sample 24

(a)

$$
\begin{aligned}
& \text { Sample } 13 \\
& \text { Sample } 14 \\
& \text { Sample } 15 \\
& \text { Sample 2 }
\end{aligned}
$$

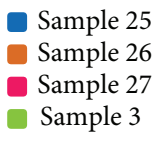

(b)

FIGURE 6: Evolution of the absorption spectra for mixtures of (a) $10 \mathrm{~nm}$ and $55 \mathrm{~nm} \mathrm{Ag} \mathrm{NPs,} \mathrm{(b)} 10 \mathrm{~nm}$ and $80 \mathrm{~nm} \mathrm{Ag} \mathrm{NPs.}$ 
The authors would like to thank Dr. Witold Zielinski of Technical University of Warsaw for TEM imaging.

\section{References}

[1] E. Boisselier and D. Astruc, "Gold nanoparticles in nanomedicine: preparations, imaging, diagnostics, therapies and toxicity," Chemical Society Reviews, vol. 38, no. 6, pp. 1759-1782, 2009.

[2] M. V. Yezhelyev, X. Gao, Y. Xing, A. Al-Hajj, S. Nie, and R. M. O'Regan, "Emerging use of nanoparticles in diagnosis and treatment of breast cancer," The Lancet Oncology, vol. 7, no. 8, pp. 657-667, 2006.

[3] Y. C. Cao, R. Jin, and C. A. Mirkin, "Nanoparticles with Raman spectroscopic fingerprints for DNA and RNA detection," Science, vol. 297, no. 5586, pp. 1536-1540, 2002.

[4] J. B. Falabella, T. J. Cho, D. C. Ripple, V. A. Hackley, and M. J. Tarlov, "Characterization of gold nanoparticles modified with single-stranded DNA using analytical ultracentrifugation and dynamic light scattering," Langmuir, vol. 26, no. 15, pp. 1274012747,2010

[5] H. Li and L. Rothberg, "Colorimetric detection of DNA sequences based on electrostatic interactions with unmodified gold nanoparticles," Proceedings of the National Academy of Sciences of the United States of America, vol. 101, no. 39, pp. 14036-14039, 2004.

[6] J.-M. Nam, C. S. Thaxton, and C. A. Mirkin, "Nanoparticlebased bio-bar codes for the ultrasensitive detection of proteins," Science, vol. 301, no. 5641, pp. 1884-1886, 2003.

[7] T. A. Taton, C. A. Mirkin, and R. L. Letsinger, "Scanometric DNA array detection with nanoparticle probes," Science, vol. 289, no. 5485, pp. 1757-1760, 2000.

[8] D. J. Gorth, D. M. Rand, and T. J. Webster, "Silver nanoparticle toxicity in Drosophila: size does matter," International Journal of Nanomedicine, vol. 6, pp. 343-350, 2011.

[9] M. Korani, S. M. Rezayat, K. Gilani, S. Arbabi Bidgoli, and S. Adeli, "Acute and subchronic dermal toxicity of nanosilver in guinea pig," International Journal of Nanomedicine, vol. 6, pp. 855-862, 2011.

[10] H. J. Johnston, G. Hutchison, F. M. Christensen, S. Peters, S. Hankin, and V. Stone, "A review of the in vivo and in vitro toxicity of silver and gold particulates: particle attributes and biological mechanisms responsible for the observed toxicity," Critical Reviews in Toxicology, vol. 40, no. 4, pp. 328-346, 2010.

[11] Y. M. Mohan, K. Lee, T. Premkumar, and K. E. Geckeler, "Hydrogel networks as nanoreactors: a novel approach to silver nanoparticles for antibacterial applications," Polymer, vol. 48, no. 1, pp. 158-164, 2007.

[12] A. J. Haes, C. L. Haynes, A. D. McFarland, G. C. Schatz, R. P. Van Duyne, and S. Zou, "Plasmonic materials for surface-enhanced sensing and spectroscopy," MRS Bulletin, vol. 30, no. 5, pp. 368375, 2005.

[13] G.-N. Xiao and S.-Q. Man, "Surface-enhanced Raman scattering of methylene blue adsorbed on cap-shaped silver nanoparticles," Chemical Physics Letters, vol. 447, no. 4-6, pp. 305-309, 2007.

[14] T. Yamaguchi, T. Kaya, and H. Takei, "Characterization of cap-shaped silver particles for surface-enhanced fluorescence effects," Analytical Biochemistry, vol. 364, no. 2, pp. 171-179, 2007.
[15] O. V. Salata, "Applications of nanoparticles in biology and medicine," Journal of Nanobiotechnology, vol. 2, article 3, 2004.

[16] J. M. Zook, V. Rastogi, R. I. MacCuspie, A. M. Keene, and J. Fagan, "Measuring agglomerate size distribution and dependence of localized surface plasmon resonance absorbance on gold nanoparticle agglomerate size using analytical ultracentrifugation," ACS Nano, vol. 5, no. 10, pp. 8070-8079, 2011.

[17] S. Link and M. A. El-Sayed, "Size and temperature dependence of the plasmon absorption of colloidal gold nanoparticles," Journal of Physical Chemistry B, vol. 103, no. 21, pp. 4212-4217, 1999.

[18] M. Hu, J. Chen, Z.-Y. Li et al., "Gold nanostructures: engineering their plasmonic properties for biomedical applications," Chemical Society Reviews, vol. 35, no. 11, pp. 1084-1094, 2006.

[19] I. Piwonski, K. Soliwoda, A. Kisielewska, K. Kadziola, and R. Stanecka-Badura, "The effect of the surface nanostructure and composition on the antiwear properties of zirconia-titania coatings," Ceramics International, vol. 39, no. 2, pp. 1111-1123, 2013.

[20] R. D. Boyd and A. Cuenat, "New analysis procedure for fast and reliable size measurement of nanoparticles from atomic force microscopy images," Journal of Nanoparticle Research, vol. 13, no. 1, pp. 105-113, 2011.

[21] M. Cichomski, E. Tomaszewska, K. Kośla, W. Kozłowski, P. J. Kowalczyk, and J. Grobelny, "Study of dithiol monolayer as the interface for controlled deposition of gold nanoparticles," Materials Characterization, vol. 62, no. 3, pp. 268-274, 2011.

[22] S. K. Brar and M. Verma, "Measurement of nanoparticles by light-scattering techniques," Trends in Analytical Chemistry, vol. 30, no. 1, pp. 4-17, 2011.

[23] B. N. Khlebtsov and N. G. Khlebtsov, "On the measurement of gold nanoparticle sizes by the dynamic light scattering method," Colloid Journal, vol. 73, no. 1, pp. 118-127, 2011.

[24] Y. Dieckmann, H. Cölfen, H. Hofmann, and A. Petri-Fink, "Particle size distribution measurements of manganese-doped ZnS nanoparticles," Analytical Chemistry, vol. 81, no. 10, pp. 3889-3895, 2009.

[25] J. Grobelny, F. W. Delrio, N. Pradeep, D. Kim -I, V. A. Hackley, and R. F. Cook, "Size measurement of nanoparticles using atomic force microscopy," in Characterization of Nanoparticles Intended for Drug Delivery, S. E. McNeil, Ed., vol. 697 of Methods in Molecular Biology, pp. 71-82, Springer, 2011.

[26] H. X. He, H. Zhang, Q. G. Li, T. Zhu, S. F. Y. Li, and Z. F. Liu, "Fabrication of designed architectures of Au nanoparticles on solid substrate with printed self-assembled monolayers as templates," Langmuir, vol. 16, no. 8, pp. 3846-3851, 2000.

[27] S. Pethkar, M. Aslam, I. S. Mulla, P. Ganeshan, and K. Vijayamohanan, "Preparation and characterisation of silver quantum dot superlattice using self-assembled monolayers of pentanedithiol," Journal of Materials Chemistry, vol. 11, no. 6, pp. 1710-1714, 2001.

[28] H. Jans, X. Liu, L. Austin, G. Maes, and Q. Huo, "Dynamic light scattering as a powerful tool for gold nanoparticle bioconjugation and biomolecular binding studies," Analytical Chemistry, vol. 81, no. 22, pp. 9425-9432, 2009.

[29] B. G. Zanetti-Ramos, M. B. Fritzen-Garcia, C. S. de Oliveira et al., "Dynamic light scattering and atomic force microscopy techniques for size determination of polyurethane nanoparticles," Materials Science and Engineering C, vol. 29, no. 2, pp. 638640, 2009.

[30] M. Zimbone, L. Calcagno, G. Messina, P. Baeri, and G. Compagnini, "Dynamic light scattering and UV-vis spectroscopy of 
gold nanoparticles solution," Materials Letters, vol. 65, no. 19-20, pp. 2906-2909, 2011.

[31] D. K. Bhui, H. Bar, P. Sarkar, G. P. Sahoo, S. P. De, and A. Misra, "Synthesis and UV-vis spectroscopic study of silver nanoparticles in aqueous SDS solution," Journal of Molecular Liquids, vol. 145, no. 1, pp. 33-37, 2009.

[32] E. Hao, G. C. Schatz, and J. T. Hupp, "Synthesis and optical properties of anisotropic metal nanoparticles," Journal of Fluorescence, vol. 14, no. 4, pp. 331-341, 2004.

[33] J. A. Creighton and D. G. Eadon, "Ultraviolet-visible absorption spectra of the colloidal metallic elements," Journal of the Chemical Society, Faraday Transactions, vol. 87, no. 24, pp. 38813891, 1991.

[34] W. Tscharnuter, "Photon correlation spectroscopy in particle sizing," in Encyclopedia of Analytical Chemistry, R. A. Meyers, Ed., pp. 5469-5485, John Wiley \& Sons, Chichester, UK, 2000.

[35] D. D. Evanoff Jr. and G. Chumanov, "Synthesis and optical properties of silver nanoparticles and arrays," ChemPhysChem, vol. 6, no. 7, pp. 1221-1231, 2005.

[36] D. E. Koppel, "Analysis of macromolecular polydispersity in intensity correlation spectroscopy: the method of cumulants," The Journal of Chemical Physics, vol. 57, no. 11, pp. 4814-4820, 1972.

[37] B. J. Berne and R. Pecora, Dynamic Light Scattering: With Applications to Chemistry, Biology, and Physics, Dover, New York, NY, USA, 2000.

[38] A. B. Leung, K. I. Suh, and R. R. Ansari, "Particle-size and velocity measurements in flowing conditions using dynamic light scattering," Applied Optics, vol. 45, no. 10, pp. 2186-2190, 2006.

[39] X. Huang, P. K. Jain, I. H. El-Sayed, and M. A. El-Sayed, "Gold nanoparticles: interesting optical properties and recent applications in cancer diagnostics and therapy," Nanomedicine, vol. 2, no. 5, pp. 681-693, 2007.

[40] R. Sato-Berŕu, R. Redón, A. Vázquez-Olmos, and J. M. Saniger, "Silver nanoparticles synthesized by direct photoreduction of metal salts. Application in surface-enhanced Raman spectroscopy," Journal of Raman Spectroscopy, vol. 40, no. 4, pp. 376380, 2009.

[41] R. D. Boyd, S. K. Pichaimuthu, and A. Cuenat, "New approach to inter-technique comparisons for nanoparticle size measurements; using atomic force microscopy, nanoparticle tracking analysis and dynamic light scattering," Colloids and Surfaces A, vol. 387, no. 1-3, pp. 35-42, 2011.

[42] C. M. Hoo, N. Starostin, P. West, and M. L. Mecartney, "A comparison of atomic force microscopy (AFM) and dynamic light scattering (DLS) methods to characterize nanoparticle size distributions," Journal of Nanoparticle Research, vol. 10, no. 1, pp. 89-96, 2008.

[43] T. Dadosh, "Synthesis of uniform silver nanoparticles with a controllable size," Materials Letters, vol. 63, no. 26, pp. 22362238, 2009.

[44] B. J. Messinger, K. U. Von Raben, R. K. Chang, and P. W. Barber, "Local fields at the surface of noble-metal microspheres," Physical Review B, vol. 24, no. 2, pp. 649-657, 1981.

[45] N. G. Khlebtsov, "Determination of size and concentration of gold nanoparticles from extinction spectra," Analytical Chemistry, vol. 80, no. 17, pp. 6620-6625, 2008.

[46] N. G. Khlebtsov, L. A. Trachuk, and A. G. Mel'nikov, “The effect of the size, shape, and structure of metal nanoparticles on the dependence of their optical properties on the refractive index of a disperse medium," Optics and Spectroscopy, vol. 98, no. 1, pp. 77-83, 2005.

[47] J.-L. Fraikin, T. Teesalu, C. M. McKenney, E. Ruoslahti, and A. N. Cleland, "A high-throughput label-free nanoparticle analyser," Nature Nanotechnology, vol. 6, no. 5, pp. 308-313, 2011. 

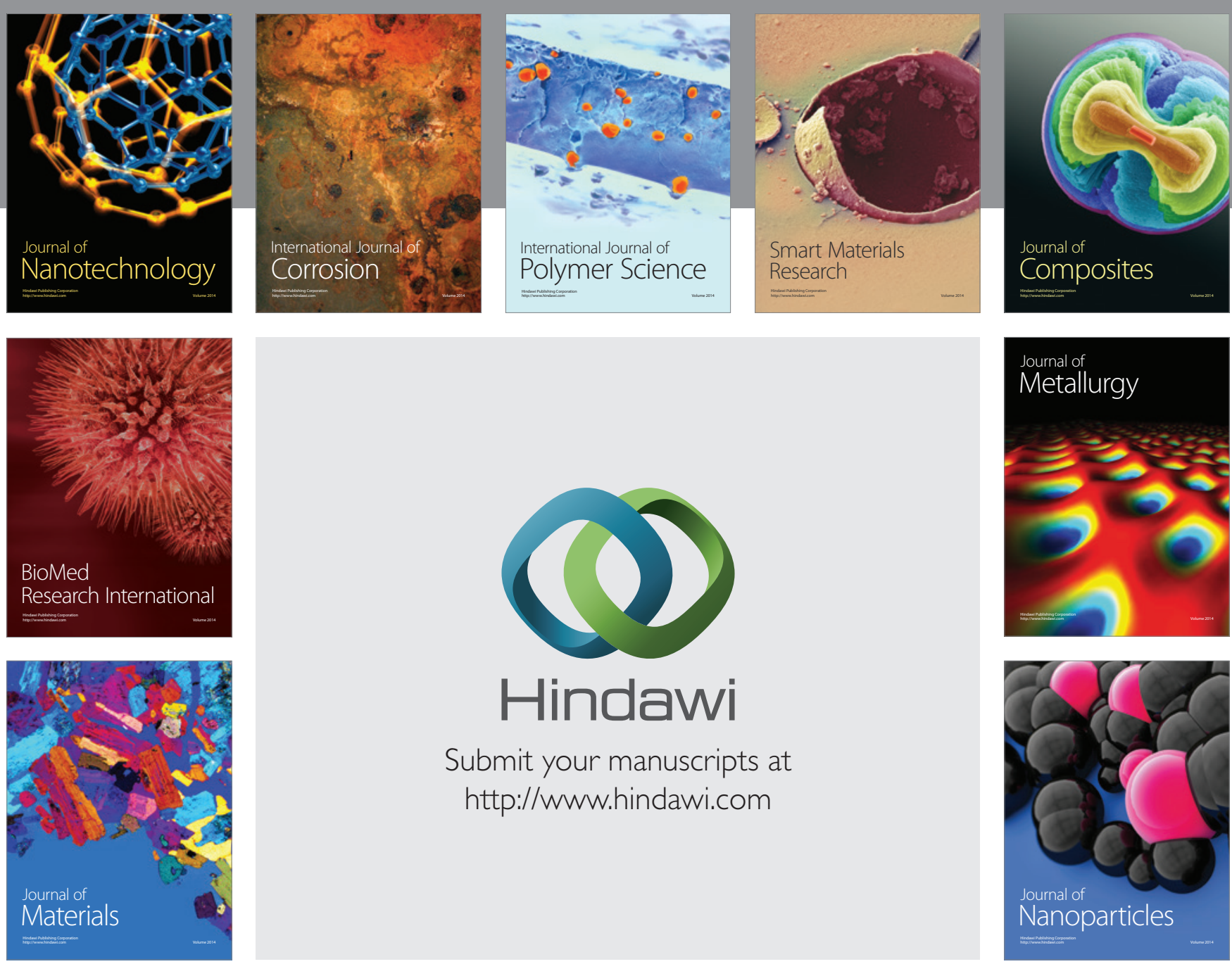

Submit your manuscripts at http://www.hindawi.com
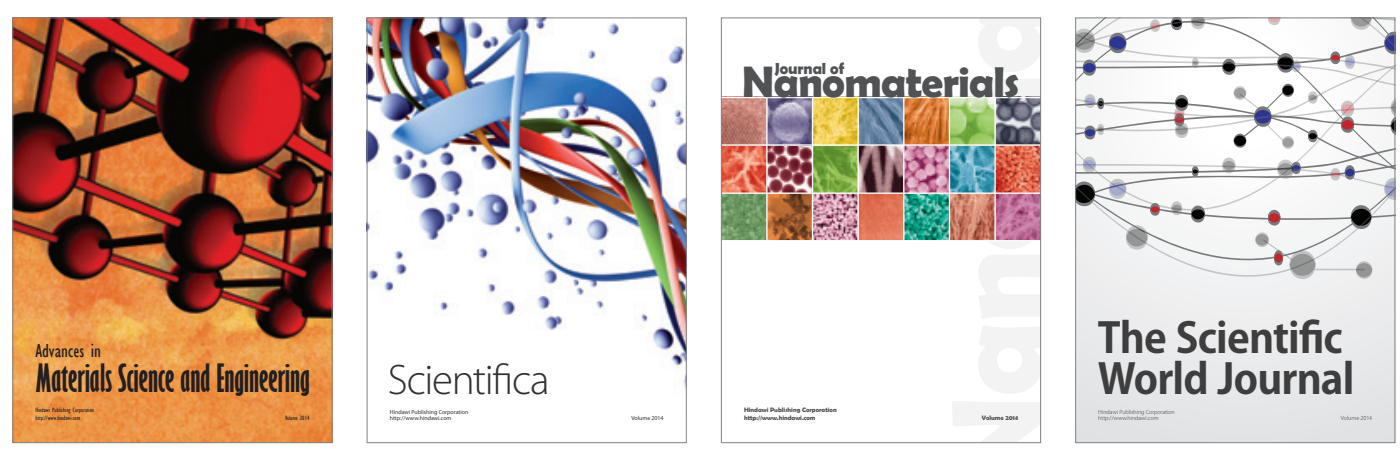

\section{The Scientific World Journal}
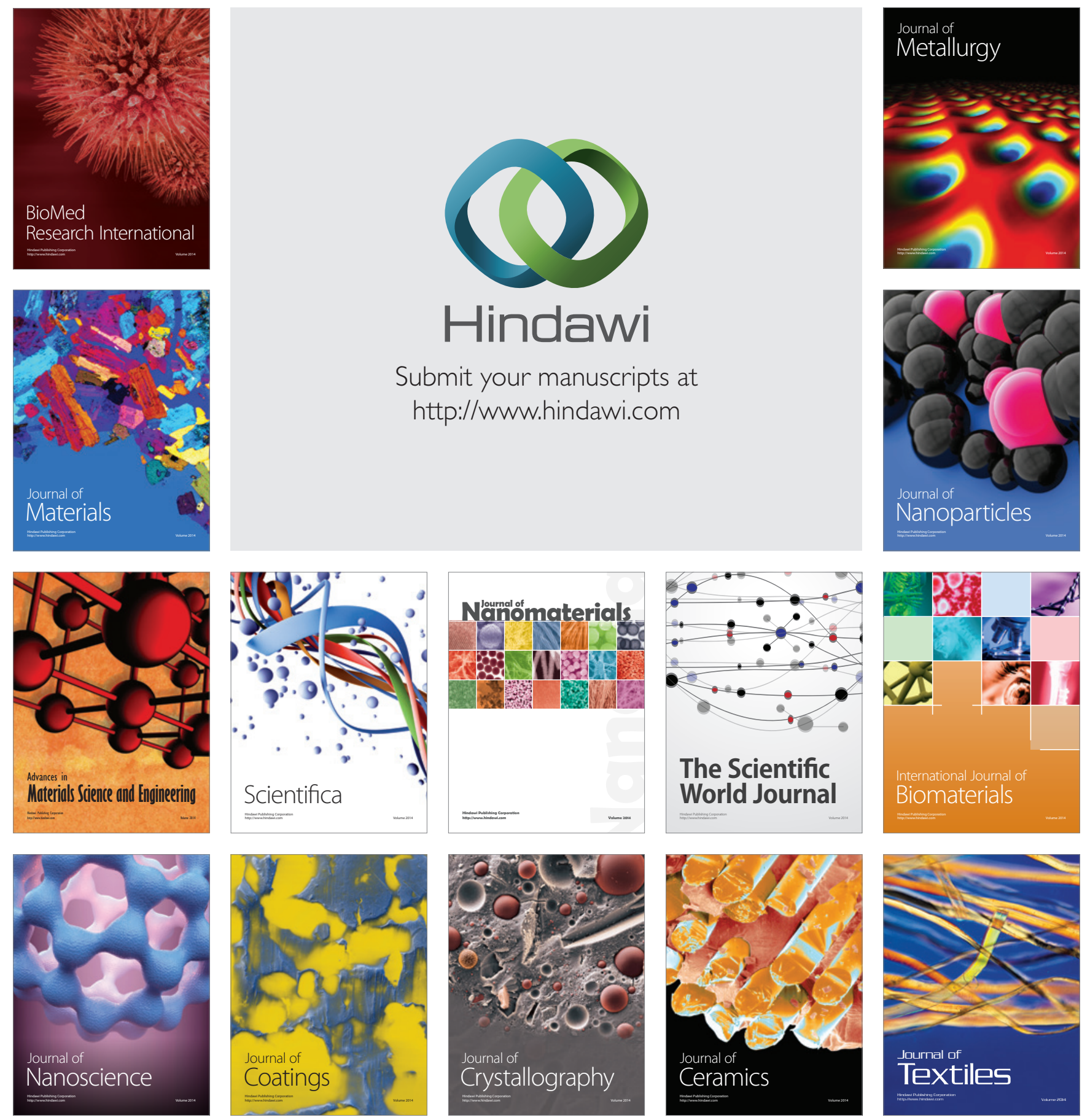